\title{
Using Augmented Reality to Mitigate Blind Spots in Trucks
}

\section{Persson, Dan Roland; Servizi, Valentino; Hansen, Tanja Lind; Bækgaard, Per}

Published in:

$\mathrm{HCl}$ in Mobility, Transport, and Automotive Systems. Automated Driving and In-Vehicle Experience Design

Link to article, DOI:

10.1007/978-3-030-50523-3_27

Publication date:

2020

Document Version

Peer reviewed version

Link back to DTU Orbit

Citation (APA):

Persson, D. R., Servizi, V., Hansen, T. L., \& Bækgaard, P. (2020). Using Augmented Reality to Mitigate Blind Spots in Trucks. In H. Krömker (Ed.), $\mathrm{HCl}$ in Mobility, Transport, and Automotive Systems. Automated Driving and In-Vehicle Experience Design (pp. 379-392). Springer. Lecture Notes in Computer Science Vol. 12212 https://doi.org/10.1007/978-3-030-50523-3_27

\section{General rights}

Copyright and moral rights for the publications made accessible in the public portal are retained by the authors and/or other copyright owners and it is a condition of accessing publications that users recognise and abide by the legal requirements associated with these rights.

- Users may download and print one copy of any publication from the public portal for the purpose of private study or research.

- You may not further distribute the material or use it for any profit-making activity or commercial gain

- You may freely distribute the URL identifying the publication in the public portal 


\title{
Using augmented reality to mitigate blind spots in trucks
}

\author{
Dan Roland Persson ${ }^{1,3}$, Valentino Servizi ${ }^{2}$, Tanja Lind Hansen ${ }^{1}$, and Per \\ Bækgaard $^{1}$ \\ 1 Department of Applied Mathematics and Computer Science, Technical University \\ of Denmark \\ $\{$ danrp,pbga\}@dtu.dk \\ 2 Machine Learning for Smart Mobility Group, Technical University of Denmark \\ valse@dtu.dk \\ 3 Corresponding author danrp@dtu.dk
}

\begin{abstract}
This paper describes the implementation, testing and benchmarking of a new augmented reality prototype that gives drivers simulated direct vision, removing blind spots directly where they are present. Using augmented reality glasses and cameras we created a prototype that could effectively make parts of the truck see-through using augmented reality panels in space relative to the truck. We compare the performance of this prototype against the current standard European blind-spot mirror solution, in terms of not only judgement errors but also dangerous situations and task loads. The comparison was done on the basis of a within-subject experiment focused on right hand turning. Test results showed significantly fewer judgement errors and dangerous situations for the AR prototype when compared to mirrors, however at the cost of a slightly higher cognitive load and stress. We believe this could be caused by a learning curve difference between AR and mirrors for the professional drivers who made up our study participants. Despite the higher loads, participants perceived the AR as covering the blind spots well.
\end{abstract}

The final authenticated version is available online at https://doi.org/ 10.1007/978-3-030-50523-3_27

Keywords: Augmented reality · Truck blind spots · User experience . Performance benchmarking · Human-computer interaction

\section{Introduction}

A unique trait of Augmented Reality (AR) is that it allows the display of data in the real world in a fundamentally different way than previously possible [16]. This allows us to alter reality in ways not possible with physical devices, with several advantages as well as disadvantages. It allows virtual objects to be embedded more or less seamlessly into the perceived world, but at the same time it can also obstruct the existing perception of reality, and in some cases be distracting to users [9]. 
In the automotive industry, AR is already used for the design and production of cars [11]. Several use cases have also been suggested in the form of heads-up displays in windshields, distance indicators, and GPS tracking to name a few [10]. The use case explored in this paper uses AR in an attempt to mitigate blind spots in heavy goods vehicles.

Blind spots [27] in trucks have long been a problem that annually continues to cost lives on roads [8]. Perhaps the most well known blind spot problem in Denmark is related to the often deadly right-hand turn $^{1}$ accidents [28]. While many solutions $^{2}[31,21]$ have been created to address blind spots, along with campaigns and policies (such as the European Union Directive 2007/38/EC [29]), these initiatives only show a modest impact in recent years [8].

This paper describes the application, design and initial tests of AR used to mitigate blind spots in trucks. While similar technology has been used in other application domains such as the F35 program [14], it has to our knowledge not been evaluated in trucks. Firstly, we present a section on related work and technological use. Second, we look at our prototype design, experimental method, and results. Finally, we discuss the possible implications and limitations of our results.

The presented AR solution is partly based on the concept of direct vision [23], which shows significant benefits and advantages over indirect sightline based solutions [17]. The proposed AR implementation, using AR glasses enables vision where the driver previously had no vision, through any opaque part of the cockpit, thereby providing a vital advantage without occluding existing critical vision [9].

\section{Related work}

Related works can be seen in terms of two main areas of interest, augmented reality in vehicles, and alternative solutions to the blind spot problem.

\subsection{AR in vehicles}

$\mathrm{AR}$ is part of the virtuality continuum [16] that covers mixed reality and refers to real-world environments being 'augmented' by virtual means, using computer graphics. AR, therefore, allows for the creation of many solutions that overlap and interact with the real world in new ways. AR is already used in many different fields and contexts; Dey et al. [4] provides an overview. AR in vehicles has been around for many years with different applications; J. L. Gabbard et al. [9] suggests 4 areas of opportunities for AR to support driver tasks:

Firstly, it can be used in heads-up displays or HUDs in the windshield. HUDs can display information without forcing drivers to divert attention away from

\footnotetext{
${ }^{1}$ Or more generally, right turn in right-handed traffic, left turn in left-hand traffic

2 https://www. continental-automotive.com/en-gl/Trucks-Buses/ Vehicle-Chassis-Body/Advanced-Driver-Assistance-Systems/ Camera-Based-Systems/ProViu-360 [Online; accessed 28-January-2020]
} 
the road by looking down at a dashboard. This also allows highlighting and supporting the driver in various other ways, such as directing the driver's attention towards sources of danger [30]. Secondly, we may use AR in wayfinding and navigation tasks, presenting routing information and guiding the user towards his destination, by overlaying routing information directly onto the road [20]. Thirdly, it can be used for driver safety and information, such as lane changing, different types of alerts and other safety systems [22]. Finally, there is an opportunity to integrate vehicle-based AR into the city-scape, both geographically as well as socially, allowing displays of information, people and places of importance to the driver [24].

Given that our solution presents the driver with safety information our solution can be classified mainly as being part of the third category. The challenges of AR in traffic situations become evident especially in the second area of opportunities, as AR brings the possibility of occluding the user's existing vision [9, 19]. When designing any AR system for traffic it is of course critically important that existing objects are not covered by virtual ones in such a way that the user might miss otherwise critical information. Hence the key aspect of successful implementations of AR in safety-critical situations is to balance informational display and occluding user vision [19]. It is likewise important to balance the cognitive load of any additional tasks and information presented to users in order not to potentially negatively impact the performance of the driver $[5,6]$.

Other applications for safety in vehicles have been proposed, examples of which are heads-up displays presenting drivers with information to improve awareness and response time in different conditions [3] or collision warning systems [18].

\section{$2.2 \quad$ Blind spots}

Blind spots in trucks, i.e. areas outside the truck where the driver cannot see, are usually covered by mirrors. The extent of these blinds spots are traditionally determined either through a step by step approach [1] or by computer modelling [23]. The goal of computer modeling here is to measure the extent of the individual blind spots, often in meters, relative to the truck. The extent of blind spots, of course, varies greatly depending on the make and model of the truck in question, the best of which might be only a few centimeters, whereas for others it may be several meters [26]. The current legal standard in the European Union was introduced in 2007, which saw requirements for mirrors increasing substantially; however, despite this, accidents continue to happen at approximately the same rate $[7,8]$. Alternative solutions have also been introduced in recent years, including different camera/monitor solutions, birds-eye views and more intelligent systems such as pedestrian tracking cameras [31,21].

The use of augmented reality to remove blind spots is in itself not a new idea. It was conceptually explored by the BMW Group for use in their Mini Coopers back in 2015 [2] and the concept has been successfully explored in other

application domains, such as with the F-35 Fighter Program [14]. However, to our knowledge, the technology has yet to be properly evaluated in trucks. 


\section{$3 \quad$ Research questions \& hypothesis}

As we have not found any research exploiting AR to reduce blind spots in trucks, we propose an AR solution based on glasses, and we wish to benchmark the performance of our system versus the standard mirror solution.

We believe a key advantage of the AR system is that it can effectively provide simulated direct sightlines to the driver, which has previously shown great promise in reducing error rates [17]. These direct sightlines increase the driver's field of view, which could assist the task of orientation [25] leading to a reduced cognitive load, thereby providing a critical advantage in some driving situations [13].

Therefore, the study looks at the performance of the proposed system, and measures error rates, cognitive load, and perceived usefulness. Our research hypotheses for the study are the following.

1. The error rate are lower when using the AR system compared to using mirrors.

2. The cognitive load is lower when using the AR system compared to using mirrors.

3. The user believes the AR system can effectively remove blind spots.

The error rate consists of two things: Judgement errors and dangerous situations (both recorded by the observer in the truck). Judgement errors are cases in which the driver completed a turn without noticing a person. Dangerous situations are situations in which a turn is initiated before correct judgement is given, expressions of doubt from participants, situations in which the participant changes their initial answer during the execution of the turn, or instances of prototype errors causing an inconsistency between the experiment setup and displayed data. Judgement errors and dangerous situations are in this case mutually exclusive, this is in order to differentiate between actual errors and possible errors i.e. potentially dangerous situations.

\section{Implementation}

The AR prototype consists of a pair of AR glasses, a computer, cameras, and an inertial measurement unit (IMU). The AR glasses in use are Dreamworld AR glasses ${ }^{3}$, which have a 90-degree field of view, $2.5 \mathrm{k}$ resolution and a built-in IMU with 3 degrees of freedom.

The prototype implementation streams the view from cameras, placed outside the truck covering blind spots, to the computer which handles basic processing and the display of the scene via the connected AR glasses. A sketch of the used system can be found in Fig. 1.

${ }^{3}$ https://www .dreamworldvision.com/product-page/dreamglass-headset [Online; accessed 28-January-2020] 


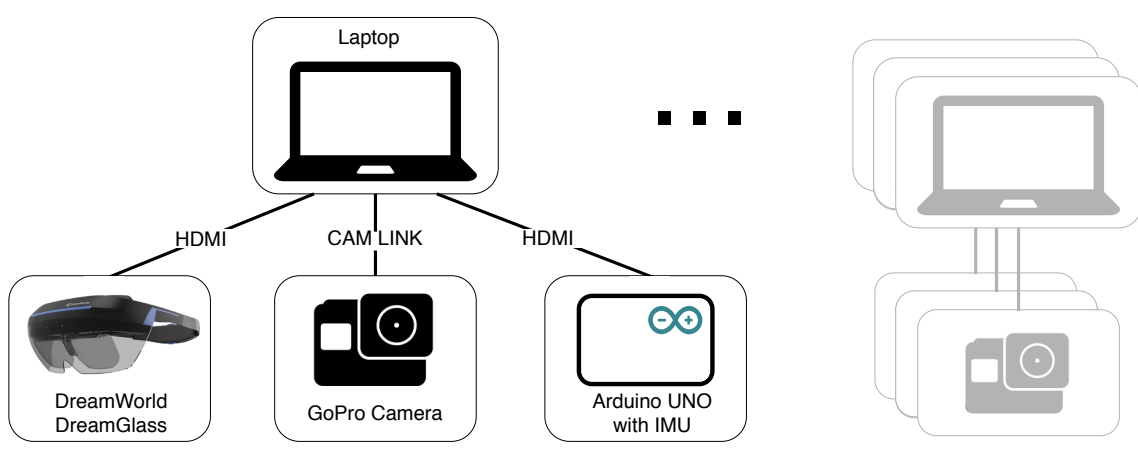

Fig. 1. Diagram of the implemented AR prototype.

Drawing inspiration from the interactions in normal cars, the augmentation of the real world is done via virtual AR panels that are placed in relevant locations in space relative to the interior of the truck, directly where blind spots are present. This creates the illusion that drivers can see through their truck, allowing them to naturally check, whenever in doubt, what is behind each blind spot. This could in the future allow drivers an overview of traffic situations without distortion, 1:1 with reality.

To avoid occluding the participants' view [9], augmented reality panels are limited to areas where the user has no existing direct vision from the driver's seat. Thus, our AR panels do not overlap areas of direct sight but rather provide vision where none was previously present. See Fig. 2.

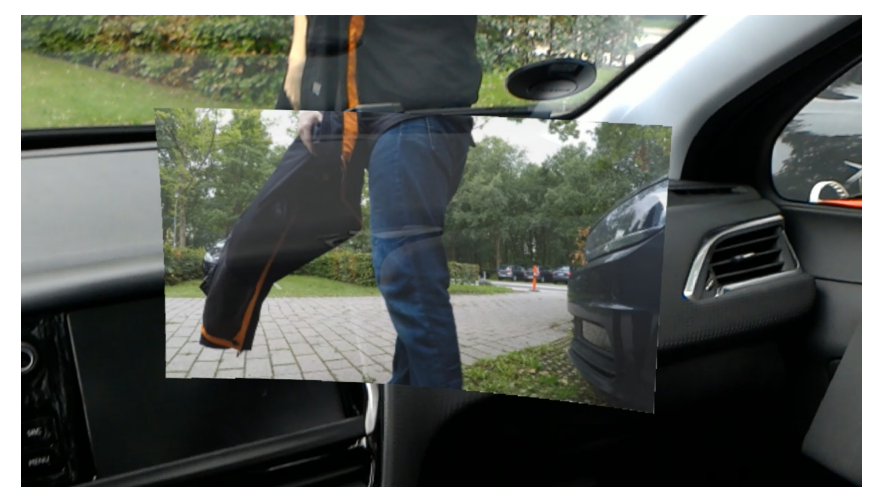

Fig. 2. An example of an AR panel in space, approximately as it will be perceived by the driver (the AR panel being transparent in our implementation).

The external IMU, seen in Fig. 1, is used to maintain the position of the AR panels relative to the interior of the truck, as relying only on the IMU in the glasses would not allow to distinguishing head movements from vehicle turning motions. The minute difference in timestamps and accuracy of IMUs 
can overtime generate some inaccuracy (or drift), which may add noise and can be a confounding factor.

\section{$5 \quad$ Method}

The study follows a within-subject approach in which participants are exposed to two levels of treatment, Mirrors or AR, represented by the independent variable Orientation type. Participants are divided into two groups being presented with either AR or mirrors first, based on the Latin square principle [15], which is used to balance any learning curve differences. The dependent variables are the Error rates (Judgment errors and Dangerous situations), Cognitive load and User acceptance, in accordance with the research question. See Table 1 for a summary. This setup allows us to benchmark the new technology against the

Table 1. Overview of experiment variables.

\begin{tabular}{|l|l|l|}
\hline Dependant variables & Independent variables & Confounding variables \\
\hline Judgement errors & Orientation (Mirrors vs AR) & Image drifting in space \\
Dangerous situations & & \\
Cognitive load & & \\
User acceptance & & \\
\hline
\end{tabular}

current standard. A confounding factor we expect will be adding noise to our experiment is AR panel drifting in the prototype, which happens due to inaccuracy between the internal and external IMU of the AR prototype. This technical limitation of our setup emerges after extended use and causes the panels to shift slightly relative to the initial position.

Cognitive load is measured through a questionnaire based on a modified and translated NASA Task Load Index [12]. We include Mental Demand, Physical Demand, and Frustration. We substitute Performance with the more specific Confidence of making a turn, and Effort with the similarly more specific Drive Difficulty and Drive Complexity. We leave out Temporal Demand, as we do not focus on temporal aspects. All questions use a 1-7 scale for which 1 is easy and 7 hard.

User acceptance is measured through an additional question for both levels, which deals with Perceived Blind Spot Coverage (rated 1-7, lower is better). Furthermore, we also ask whether AR is perceived as covering the blind spots (Yes/No).

\subsection{Equipment \& setup}

The experiment is conducted on a test track of an approximate 50 by 50 meters closed area marked by traffic cones. The track consists of 4 right-hand turns, two of which are obstructed from direct view in the driving direction using parked 


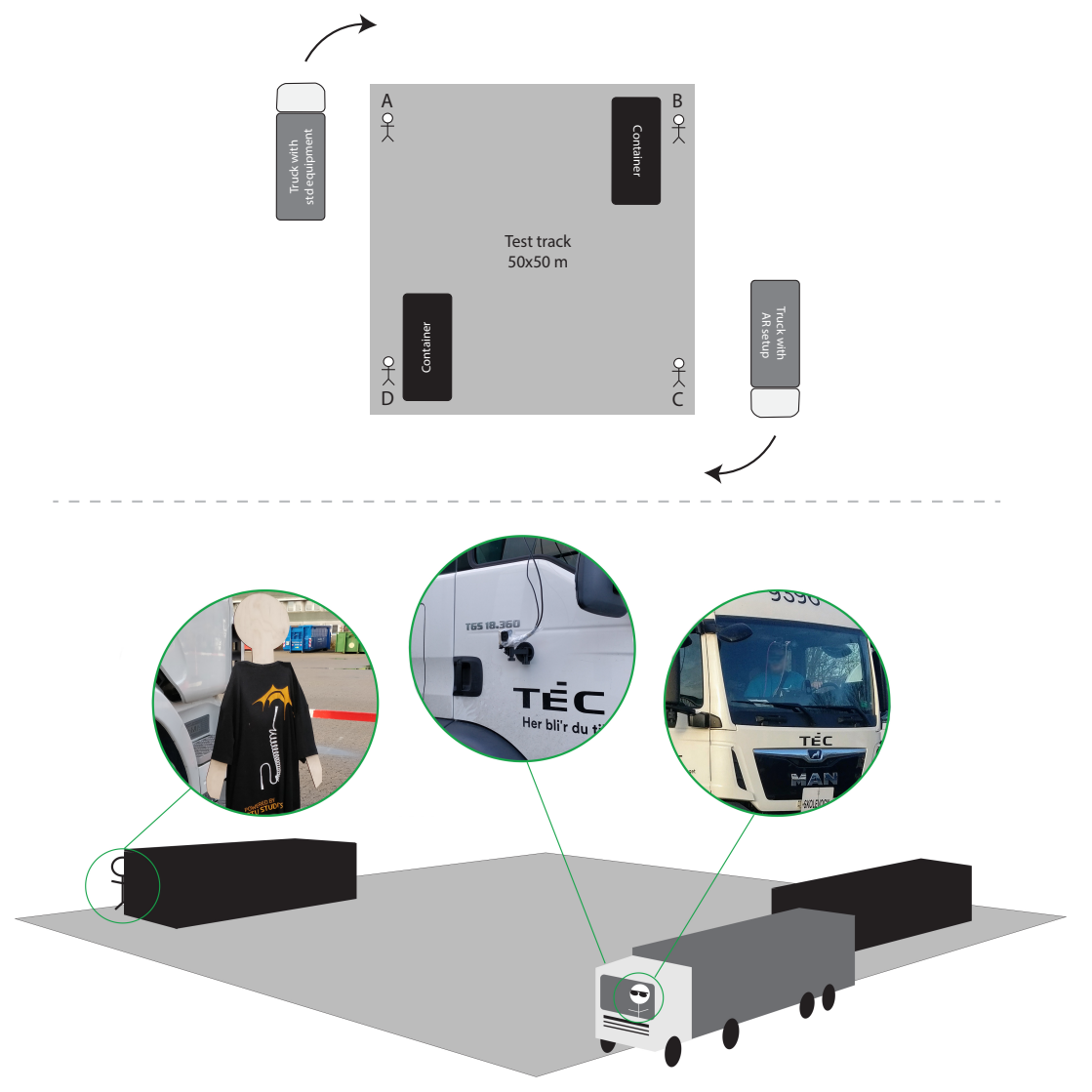

Fig. 3. Above is a bird's-eye view of the test track with equipment annotations and track dimensions. Below an illustration of the AR and experiment set-up with images (test dummy, exterior-mounted camera, driver with AR glasses) taken during the test.

trucks. At each corner of the track, denoted A-B-C-D, a person or a test dummy can be present. A visualization of the track can be seen in Fig. 3.

The experiment is performed using two similar trucks, one equipped with the standard European blind spot mirror setup and the other additionally equipped with our custom made AR system.

\subsection{Task \& procedure}

Initially, participants are introduced to the experiment and a consent form is reviewed and signed, followed by a short demographic questionnaire. Participants then enter the truck and are allowed to adjust relevant controls. Those starting with the AR level are introduced to the AR system before driving and can instruct the observer to help adjust the system if needed. Participants that use AR later will be instructed similarly. 
When ready the participant begins to drive around the test track. During each turn, the participant is asked by the observer inside the truck whether or not a person is present in the turn, the answer to which is recorded manually by the observer. This method is repeated until all 4 turns of the lap are completed. When the driver finishes a lap, the observer instructs the driver to stop for a few seconds, while the next lap is prepared and test dummies are moved around. Once the test personnel finishes the setup the driver can begin the next lap. This is repeated until all 5 laps are completed. To measure the workload of the tasks given, the previously mentioned task load questionnaire is used. Therefore, once all laps are completed, the participant is given a quick questionnaire. The next level (Mirrors or AR) is then introduced and started, performed in the same manner as the previous one. Upon completion of the second level, the participants have finished all the tasks of the experiment. Overall, each experiment takes approximately 30 minutes per participant.

Test dummies are moved around between each lap of the test track varying in number and location. 0-4 test dummies are present in any one lap, the order of which was randomized during the design of the experiment. At each level, for consistency, all drivers are subjected to the same number and relative ordering of dummies present throughout the experiment.

\subsection{Participants}

In total, the 15 participants complete 90 laps with $\mathrm{AR}$ and 85 with mirrors. The final data set consists of data from 12 males and 3 females with the average participant having more than 20 years of driving experience and an average age of 50. The full dataset contains judgement errors, dangerous situations, demographic data, task loads, and user acceptance data in the form of questionnaires.

Overall, 16 participants volunteered as test subjects in the study, all recruited by Danish Transports and Logistics (DTL) and the drivers union 3F through their respective memberships. However, due to illness before the start of the experiment, 1 participant dropped out at the last minute. Further, 3 participants had to leave the experiment early due to time constraints and 1 due to motion sickness possibly induced by the AR system. The 4 participants who did not fully finish the experiment have been included for completeness. Additionally, 4 other participants accomplished double-length experiments with twice the number of laps for both levels, due to their availability.

No participants have previous experience with the AR system in trucks and limited experience (if any) with AR.

\section{Results}

As the experiment is within-subject, either a paired Student's T-test or a Wilcoxon signed-rank test is performed depending on whether or not the data is normally distributed, to test whether any differences between the AR and Mirror levels are significant or not. We use a $p$-value $<0.05$ to signify statistical significance. 


\subsection{Judgement errors \& dangerous situations}

The judgment errors and dangerous situations, for both AR and mirrors can be seen in Fig. 4. The average error rate is $1.0(\sigma=1.50)$ errors per participant using mirrors and $0.4(\sigma=1.01)$ for AR. Dangerous situations average at 2.00 $(\sigma=1.75)$ and $0.33(\sigma=1.01)$, for mirrors and AR respectively. The differences between the levels (AR/Mirror) are statistically significant (Wilcoxon $W_{\text {errors }}=$ $30, p<0.05$ and $\left.W_{\text {dangerous }}=55, p<0.01\right)$.
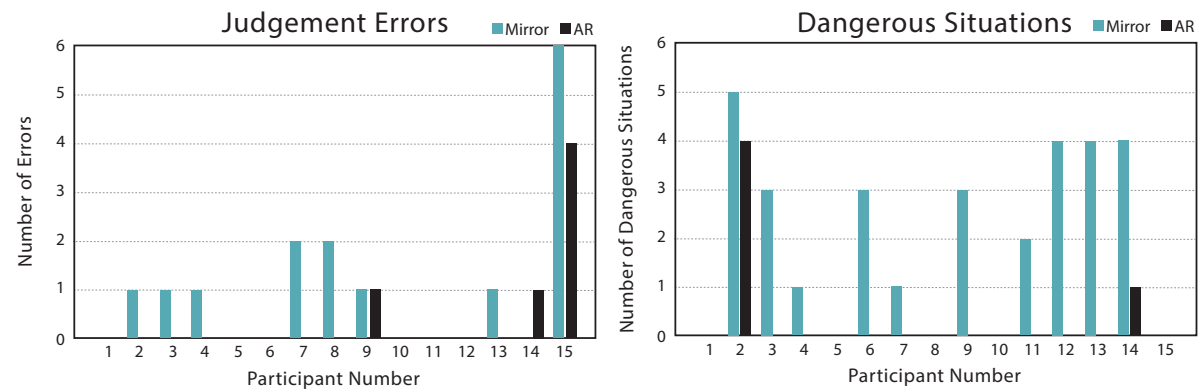

Fig. 4. Total number of judgement errors \& dangerous situations per person $(n=15)$, with blue representing mirrors and black AR.

\subsection{Task loads}

Participants rate the Mental Demand at an average of 3.6 for AR and 2.33 for mirrors, the difference is statistically significant, $(p=0.007)$. The distribution can be seen in Fig. 5. Participants rate the Frustration at an average of 3.27 for $\mathrm{AR}$ and 2.13 for mirrors, the difference is statistically significant, $(p=0.039)$. The distribution can be seen in Fig. 6 .
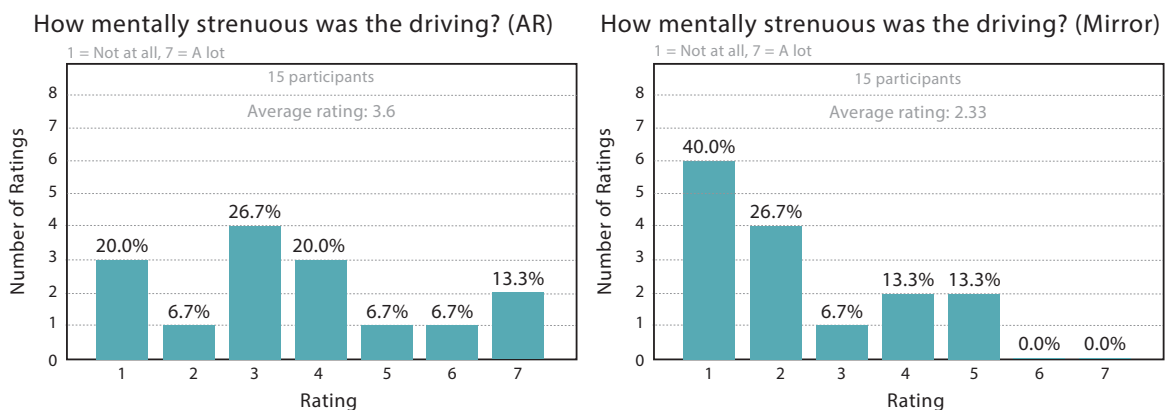

Fig. 5. Comparison between the AR and the mirror solutions measuring the Mental Demand of each solution. 

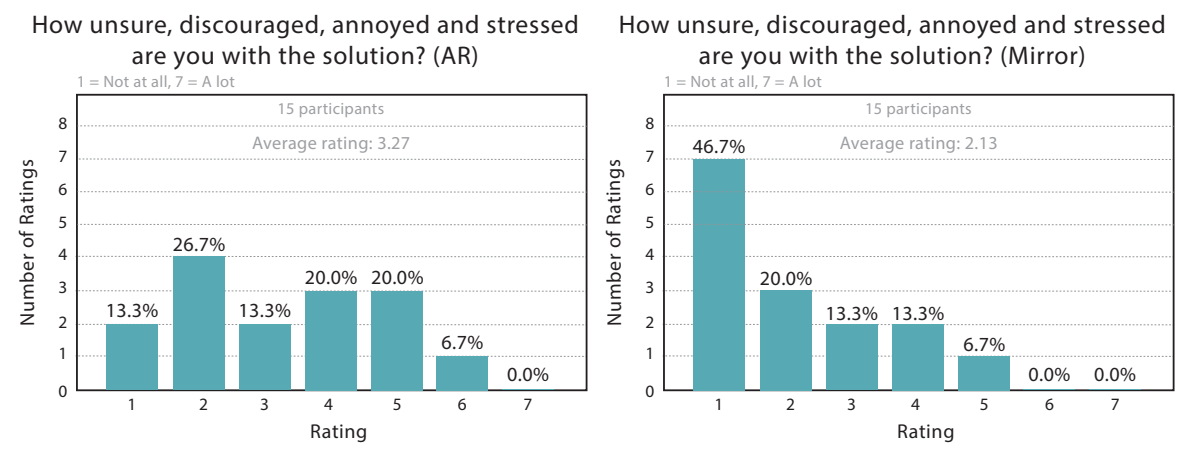

Fig. 6. Comparison between the AR and the mirror solutions measuring the Frustration of each solution.

Participants rate the perceived Physical Demand at an average of 2.67 for AR and 2.27 for mirrors, the difference is not statistically significant. Participants rate the Drive Complexity at an average of 2.8 for AR and 2.1 for mirrors, the difference is not statistically significant. Participant Confidence is likewise not statically significant, with an average of 2.93 for AR and 2.4 for mirrors. Participants rate the Drive Difficulty at an average of 2.53 for AR and 2.07 for mirrors, the difference is not statistically significant.

\subsection{User acceptance}

In the questionnaires given to participants after each level, they were also asked to rate the perceived coverage of the blind spot, on a scale from 1 to 7 , similarly to the scale used for the task loads, 1 being the best score, and 7 being the worst. The results showed AR having an average user acceptance score of 2.47 while mirrors had an average score of 3.87. Interestingly this could hint that AR might be perceived to cover the blind spots better than mirrors despite the difference not being statistically significant $(\mathrm{p}=0.1)$.

Fig. 7 shows an interesting trend, in the Yes/No question for the AR level: Every participant that started with the mirror level felt AR was properly covering the blind spots, while only $28 \%$ of the AR first group did the same. Overall $2 / 3^{r d s}$ of participants rated AR as covering the blind spots. This could indicate that drivers who started with the mirror level found tasks significantly easier to perform when using the AR solution, while those who started with AR had no initial comparative basis.

\section{Discussion}

In the present study, the relative performance at right-hand turning for AR and mirrors is compared in terms of both judgement errors, dangerous situations, cognitive load, and perceived usefulness. 

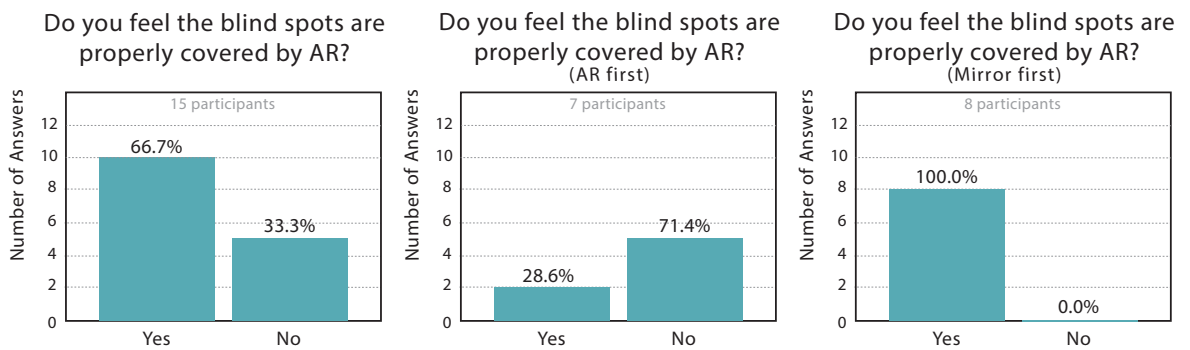

Fig. 7. Comparison of answers given to whether blind spots are perceived as covered by AR, for all participants (left) and divided by the first level presented (mid and right).

Looking at the proposed hypotheses, starting with hypothesis 1, "The error rate is lower when using AR", we find that our experiments support this by showing significantly lower judgement errors and dangerous situations. Even if AR may have suffered from technical issues, mirrors may have likewise performed less than optimally, as not all truck drivers adjusted the mirrors to suit them before starting the test, thus causing both to perform less than optimally.

Hypothesis 2, "The cognitive load is lower when using the AR system", cannot be supported given that the results of our task load questionnaire assessment show a significantly worse performance of the AR in terms of Frustration and perceived Mental demand. However, we speculate that this could be due to the learning curve difference of a novel AR system;.Drivers have years of experience with mirrors, but not with AR. This new hypothesis, however, requires further testing geared towards minimizing or removing the learning curve difference. One such test might include long term testing or repeating the experiment on truck driving students.

Concerning hypothesis 3, "The user believes the AR solution can effectively remove blind spots", while supported by a majority (two thirds) of the users perceiving AR to cover blind spots, the difference in user acceptance rating between AR and mirrors is not statistically significant. The results are interesting given that the drivers have little to no experience with AR other than what they experienced during the tests, and despite that, they rated the AR system positively. Even though we cannot claim any significance without further testing, we may have indications in support of this theory. Interestingly, all users in the mirror first group perceived AR as covering the blind spots. This consensus could indicate that those starting with mirrors found the given tasks easier to complete when using the AR system, while those beginning with AR had no comparative basis.

Overall, it can be argued that the technology for this type of solution is not yet mature enough for real-life implementations such as the one suggested. There may be uncertainty about users' acceptance of wearing AR glasses, especially given the current size of AR glasses. Nevertheless, the study presented here seems 
to align well with an understanding that direct vision [17] may have advantages, even when that vision is simulated.

Multiple points of improvement have been identified throughout the tests for future works, such as several prototype improvements. Firstly in terms of prototype stability. Secondly in regards to reducing AR panel drift which could be solved by additional sensor data or spatial anchors. Looking towards the limitations of the experiments in scope and complexity, future work might also include different blind spots and long term testing to (in)/validate the learning curve difference.

\section{Conclusion}

In our study, we compare the performance of mirrors and the proposed AR solution in mitigating blind spots. A significantly lower error rate is discovered for AR compared to mirrors in both numbers of judgement errors and dangerous situations.

Our tests also indicate that the reduced error rates come at a slightly higher Mental Demand and increased Frustration, identified by the task load questionnaire. This might be due to a learning curve difference between conventional mirrors and AR.

Drivers overall perceive AR as covering blind spots, although the rated user acceptance difference between AR and mirrors is not statistically significant. An observation is made towards how drivers rate $\mathrm{AR}$ as covering the blind spots: Drivers with a comparative basis rate AR to provide better coverage.

Acknowledgements. The authors would like to thank the involved members from Danish Transports and Logistics (DTL), the drivers union $3 \mathrm{~F}$ and TEC Hvidovre for their support and resources, especially in regards to participant recruitment and available test track. We are also grateful to Thomas Bjelbo Thomsen for his technical assistance and help during the execution of the study. Lastly, we would also like to thank Micro Grant and the FDE fund for providing funding for the project.

\section{References}

1. Ball, J.K., Danaher, D.A., Ziernicki, R.M.: A method for determining and presenting driver visibility in commercial vehicles. Tech. rep., SAE Technical Paper (2007)

2. Bell, K.: Bmw steps into augmented reality with ar driving glasses for mini. (2015), https://mashable.com/2015/04/19/bmw-mini-ar-driving-glasses, [Online; accessed 28-January-2020]

3. Charissis, V., Papanastasiou, S.: Human-machine collaboration through vehicle head up display interface. Cognition, Technology \& Work 12(1), 41-50 (2010)

4. Dey, A., Billinghurst, M., Lindeman, R.W., Swan, J.: A systematic review of 10 years of augmented reality usability studies: 2005 to 2014. Frontiers in Robotics and AI 5, 37 (2018) 
5. Engström, J., Johansson, E., Östlund, J.: Effects of visual and cognitive load in real and simulated motorway driving. Transportation research part F: traffic psychology and behaviour 8(2), 97-120 (2005)

6. Engström, J., Markkula, G., Victor, T., Merat, N.: Effects of cognitive load on driving performance: The cognitive control hypothesis. Human factors 59(5), 734$764(2017)$

7. European Commission: Report on implementation of directive 2007/38: retrofitting blind-spot mirrors (2016), https://ec.europa.eu/transport/road $\backslash$ _safety/sites/roadsafety/files/pdf/mirrors\_report\_2012\_en.pdf, [Online; accessed 28-January-2020]

8. European Road Safety Observatory: Annual accident report 2018. Tech. rep., European Commission (2018)

9. Gabbard, J.L., Fitch, G.M., Kim, H.: Behind the glass: Driver challenges and opportunities for ar automotive applications. Proceedings of the IEEE 102(2), 124-136 (2014)

10. Haeuslschmid, R., Pfleging, B., Alt, F.: A design space to support the development of windshield applications for the car. In: Proceedings of the $2016 \mathrm{CHI}$ Conference on Human Factors in Computing Systems. pp. 5076-5091 (2016)

11. Halim, A.A.: Applications of augmented reality for inspection and maintenance process in automotive industry. Journal of Fundamental and Applied Sciences 10(3S), 412-421 (2018)

12. Hart, S.G.: Nasa task load index (tlx) (1986)

13. Lee, Y.C., Lee, J.D., Ng Boyle, L.: The interaction of cognitive load and attentiondirecting cues in driving. Human factors 51(3), 271-280 (2009)

14. Lockheed Martin Corporation: Unprecedented situational awareness (2019), https://www.f35.com/about/capabilities/helmet, [Online; accessed 28January-2020]

15. MacKenzie, I.: Designing hci experiments. Human-computer interaction: an empirical research perspective p. 176 (2013)

16. Milgram, P., Kishino, F.: A taxonomy of mixed reality visual displays. IEICE TRANSACTIONS on Information and Systems 77(12), 1321-1329 (1994)

17. Milner, R., Williams, H.W.: Transport for london: Exploring the road safety benefits of direct vs indirect vision inhgv cabs, direct vision vs indirect vision: A study exploring the potential improvements to road safety through expanding the hgv cab field of vision. Tech. rep., Ove Arup \& Partners Ltd (2016)

18. Park, B.J., Yoon, C., Lee, J.W., Kim, K.H.: Augmented reality based on driving situation awareness in vehicle. In: 2015 17th International Conference on Advanced Communication Technology (ICACT). pp. 593-595. IEEE (2015)

19. Pärsch, N., Harnischmacher, C., Baumann, M., Engeln, A., Krauß, L.: Designing augmented reality navigation visualizations for the vehicle: A question of real world object coverage? In: International Conference on Human-Computer Interaction. pp. 161-175. Springer (2019)

20. Pfannmueller, L., Kramer, M., Senner, B., Bengler, K.: A comparison of display concepts for a navigation system in an automotive contact analog head-up display. Procedia Manufacturing 3, 2722-2729 (2015)

21. Pyykonen, P., Virtanen, A., Kyytinen, A.: Developing intelligent blind spot detection system for heavy goods vehicles. In: 2015 IEEE International Conference on Intelligent Computer Communication and Processing (ICCP). pp. 293-298. IEEE (2015) 
22. Rameau, F., Ha, H., Joo, K., Choi, J., Park, K., Kweon, I.S.: A real-time augmented reality system to see-through cars. IEEE transactions on visualization and computer graphics 22(11), 2395-2404 (2016)

23. Robinson, T., Knight, I., Martin, P., Manning, J., Eyers, V.: Definition of direct vision standards for heavy goods vehicles (hgvs). Tech. rep., Technical report RPN3680, Berkshire, TRL (2016)

24. Schroeter, R., Rakotonirainy, A., Foth, M.: The social car: new interactive vehicular applications derived from social media and urban informatics. In: Proceedings of the 4th International Conference on Automotive User Interfaces and Interactive Vehicular Applications. pp. 107-110 (2012)

25. Sieker, T.G., Skulason, T.G., Sletting, K., Trolle, T.K., Hammershøi, D.: A cognitive analysis of truck drivers' right-hand turns. Trafikdage På Aalborg Universitet (online) 2015 (2015)

26. Stef Cornelis, W.T.: Eliminating truck blind spots a matter of direct vision. Tech. rep., Transport \& Environment (2016)

27. Summerskill, S., Marshall, R., Paterson, A., Reed, S.: Understanding direct and indirect driver vision in heavy goods vehicles: Final report (2015)

28. Summerskill, S., Marshall, R., Lenard, J.: The design of category n3 vehicles for improved driver direct vision. Loughborough Design School. Report for Transport for London and Transport \& Environment (2014)

29. The European Parliament and of the council: Directive 2007/38/ecof. eu, 2007 (2007)

30. Tonnis, M., Sandor, C., Klinker, G., Lange, C., Bubb, H.: Experimental evaluation of an augmented reality visualization for directing a car driver's attention. In: Fourth IEEE and ACM International Symposium on Mixed and Augmented Reality (ISMAR'05). pp. 56-59. IEEE (2005)

31. Van Beeck, K., Goedemé, T., Tuytelaars, T.: Towards an automatic blind spot camera: robust real-time pedestrian tracking from a moving camera. In: Proceedings of the twelfth IAPR conference on machine vision applications. pp. 528-531. MVA Organization; Japan (2011) 